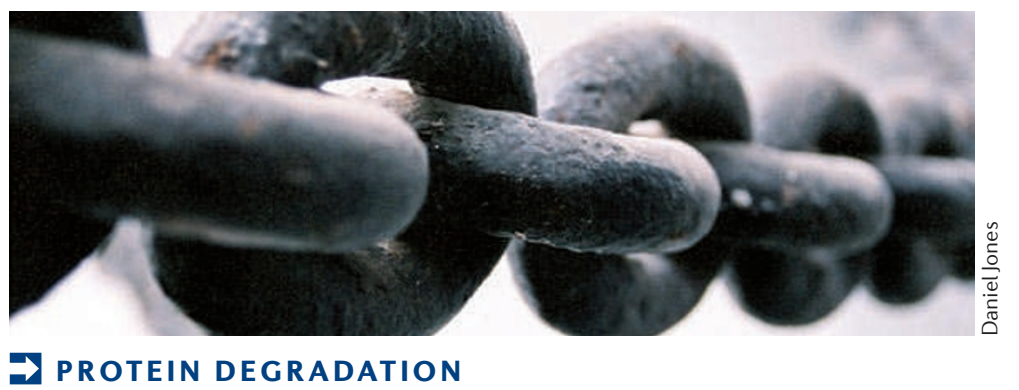

\section{Chain gang}

Ubiquitin has seven Lys residues, which all contribute to the assembly

...all non-

Lys63-linked polyubiquitin chains mediate proteasomal degradation... of polyubiquitin chains on protein substrates. Lys48-linked chains lead to protein degradation and Lys63-chains act in non-proteolytic events, but the roles of the other 'unconventional' polyubiquitin chains have been elusive. Junmin Peng and colleagues now report that all non-Lys63-linked polyubiquitin chains target proteins for proteasomal degradation. Moreover, they show that Lys11-linked chains function in endoplasmic reticulum (ER)-associated degradation (ERAD) - a quality control pathway in which misfolded or improperly assembled proteins of the ER are ubiquitylated and degraded by the $26 \mathrm{~S}$ proteasome.

Using mass spectrometry (MS) in yeast, the authors found high levels of unconventional polyubiquitin chains (especially those linked to Lys11) on a broad range of substrates. The levels of these chains, as well as of Lys48-linked chains, increased when the $26 \mathrm{~S}$ proteasome was chemically or genetically inhibited, whereas the level of Lys63-linked chains was not affected (which was to be expected, given the well-known non-proteolytic functions of Lys63linked chains). These results suggest that all non-Lys63-linked polyubiquitin chains mediate proteasomal degradation.

If the unconventional polyubiquitin chains direct proteins for degradation, the ubiquitin moieties of these chains might be recycled by the activity of deubiquitylating enzymes (DUBs) that are associated with the $26 \mathrm{~S}$ proteasome. Indeed, the authors found that all unconventional chains are cleaved by proteasome-associated DUBs in vitro.
In yeast, mutation of ubiquitin Lys48 is lethal, but Lys48 alone is not sufficient to confer viability when the other Lys residues are mutated. So, the unconventional polyubiquitin chains have important physiological functions. Notably, by combining multiple substitutions of the chain-forming Lys residues, the authors found that the unconventional chains have partially redundant functions.

Using quantitative MS, Peng and co-workers searched for Lys11 polyubiquitin chain-linked substrates in the entire proteome of wild type and a ubiquitin Lys11 mutant strain. Among other substrates, they identified Ubc6, a ubiquitin-conjugating enzyme that is involved in ERAD. Mutation of ubiquitin Lys11 significantly reduced the turnover rate of Ubc6, and Lys11 mutant cells have increased sensitivity to ER stress-inducing agents and an elevated unfolded protein response. So, Lys11-linked polyubiquitin chains function in ubiquitylation events that are related to ERAD.

Given the broad spectrum of proteins that are altered in the Lys11 mutant, Lys11-linked polyubiquitin chains are likely to be involved in many physiological processes. The specific function of the diverse ubiquitin chains in different processes might be achieved during conjugation, recognition by ubiquitin receptors and disassembly by DUBs.

Francesca Cesari

ORIGINAL RESEARCH PAPER Xu, P. et al.

Quantitative proteomics reveals the function of unconventional ubiquitin chains in proteasomal degradation. Cell 137, 133-145 (2009) FURTHER READING Ravid, T. \& Hochstrasser, M. Diversity of degradation signals in the ubiquitinproteasome system. Nature Rev. Mol. Cell Biol. 9 , 679-689 (2008) |Vembar, S. S. \& Brodsky, J. L. One step at a time: endoplasmic reticulumassociated degradation. Nature Rev. Mol. Cell Biol. 9, 944-957 (2008) 